\title{
Variación lingüística en la expresión del pretérito en el español del siglo XIX de España y México: Rasgos deícticos y aspectuales
}

\author{
Francisco Salgado Robles* \\ University of Kentucky, Estados Unidos \\ Ana María Díaz Collazos \\ University of Florida, Estados Unidos
}

\begin{abstract}
Resumen
Este trabajo estudia y analiza la variación lingüística de dos tiempos verbales -pretérito perfecto simple (PPS) y pretérito perfecto compuesto (PPC) - en la lengua española del siglo XIX de España y México. La diferenciación dialectal de los valores temporales en el español ibérico y americano ha sido tema de especial interés desde el punto de vista sincrónico en la modalidad contemporánea de la lengua. Sincrónicamente se ha destacado el proceso de gramaticalización que conlleva la pérdida del sentido léxico de haber en la formación de los tiempos compuestos. Sin embargo, el estadio entre la formación del PPC y la generalización del PPC en el español peninsular no ha sido observado. Ante la necesidad de describir esa transformación, como objetivos nos planteamos determinar si las diferencias en la
\end{abstract}

Para correspondencia, dirigirse a: Francisco Salgado (fsalgadorobles@uky.edu), University of Kentucky, Department of Hispanic Studies, 1139 Patterson Office Tower , Lexington, KY 40506 o a Ana María Díaz (anamdiaz@ufl.edu), University of Florida, Department of Spanish and Portuguese, 170 Dauer Hall, Gainesville, FL 32611. 
frecuencia de uso de ambos tiempos son paralelas en España y en América, y examinar la función temporal en el español de ambos continentes en esta época. Dos obras representativas del español del siglo XIX de España y México conforman nuestro corpus, el cual procesamos a través del programa de análisis múltiple GoldVarb. Además de las variables dependientes (PPS y PPC), consideramos seis factores lingüísticos como variables independientes. De nuestro análisis se desprende que entre México y España ya en el siglo XIX encontramos una diferencia significativa en el uso del PPS y PPC. La preferencia por la forma compuesta en el español peninsular más que en el americano indica la existencia de una variación dialectal. Nuestro trabajo revela que el sistema temporal que distingue el español peninsular en el ámbito del pretérito es un fenómeno más antiguo de lo que se cree.

Palabras clave: variación lingüística, pretérito, español del siglo XIX, español de España, español de México, deixis, aspecto.

LINGUISTIC VARIATION IN THE PRETERIT EXPRESSION IN THE 19TH

Century Spanish in Spain and Mexico: Deictic and aspectual FEATURES

\section{ABSTRACT}

This article examines and analyzes the linguistic variation of two verb tenses - present perfect (PP) and preterit (PRET) - in the 19th Century Peninsular and Mexican Spanish. Scholars have been interested in the dialectal differences of the time values in the European and American Spanish, from a synchronic angle in the contemporary stages of language. Synchronically it has been pointed out that the process of grammaticalization results in the loss of lexical sense of have in the construction of complex tenses. However, there has been no examination of the phase between the formation of PP and the generalization on the Peninsular Spanish. Given the need to describe this transformation, we are seeking to establish whether there are parallel differences in the usage frequency of both tenses in Spain and Mexico, and to examine the time function in the Spanish of both continents in this time. Our data comes from two works representing the 19th Century Spanish of Spain and Mexico, which we analyzed using GoldVarb. In addition to the dependent variables (PRET and PP), we take into account six linguistic factors as independent variables. Our analysis shows that there is a significant difference between 19th Century Mexican and Peninsular Spanish in the use of PRET and PP. The preference for the complex form in the European 


\begin{abstract}
Spanish, distinct to that of the American Spanish, indicates an existing dialectal variation. Our study reveals that the time system distinguishing the Peninsular Spanish in the preterit contour is an older phenomenon than it was believed to be.
\end{abstract}

Key words: linguistic variation, preterit, 19th Century Spanish, Peninsular Spanish, Mexican Spanish, deixis, aspect.

Recibido: 01/04/12

Aceptado: 20/05/12

\title{
1. INTRODUCCIÓN
}

Entre los estudiosos de la lingüística histórica de lenguas romances, un tema de particular interés corresponde a los cambios temporales, dentro de los cuales, según la mayoría de los estudios filológicos, encuentran de gran atracción la inestable relación entre el uso de tiempos verbales que atañen al pasado. Por esta razón, el fenómeno lingüístico aquí objeto de examen es el estudio y análisis del comportamiento sincrónico de dos tiempos de pasado en la lengua española, esto es, los rasgos deícticos y aspectuales del pretérito perfecto simple (en adelante PPS, canté) y del pretérito perfecto compuesto (en adelante PPC, he cantado) en el español del siglo XIX de España y México. Como objetivos, se intenta (i) averiguar si existen diferencias en la frecuencia de uso del PPS y el PPC en ambas variedades, (ii) determinar si existe ya un cambio en marcha, como recogemos en el repaso bibliográfico, especialmente en el español peninsular, a extender los contextos de uso del PPC, y (iii) examinar la función temporal anteriormente mencionada en el habla en ambos continentes en dicho marco temporal, según la comparación de las variables lingüísticas que puedan estar afectando el uso del pasado. El presente artículo se divide en cuatro secciones: en la primera, se hace un breve recuento de los problemas y hallazgos de la investigación que se ha realizado al respecto; la segunda presenta el sustento metodológico de la investigación; en la tercera se expresan los resultados cuantitativos con una discusión sobre los mismos. El último apartado expone las conclusiones a las que llegamos, así como sugiere futuras líneas de investigación. 


\section{ESTADO DE LA CUESTIÓN}

Siguiendo la gramática de Seco (1985), tanto el PPS (canté) como el PPC (he cantado) expresan hechos perfectos y anteriores al momento en que hablamos. Si la acción tiene "cierta cohesión temporal con el presente, entonces se empleará el PPC; si, por el contrario, la acción no cae dentro de ese presente psicológico, el tiempo usado será el PPS. Ese presente psicológico tiene una amplitud muy variable: puede abarcar sólo el instante actual o puede abarcar muchos años" (74). De este modo, en "La guerra terminó el mes pasado" el presente psicológico corresponde a este mes. Entonces, Seco se coloca en el punto de vista del hablante, en el que intervienen no solo la distancia temporal (elemento objetivo), sino también el mayor o menor interés (elemento subjetivo) que se tenga en la acción. Conforme la teoría de Alarcos (1978), el PPC indica una acción que se acerca al presente gramatical. Dicho con otras palabras, apunta que la acción ha ocurrido en el mismo periodo de tiempo en que se habla o se escribe, pero sin coincidir con el acto de hablar o escribir. No obstante, el PPS designa una acción ocurrida en el pasado con un límite en ese mismo pasado. El uso de ambos tiempos "no indica que la acción sea próxima o remota en el sentido absoluto de estas palabras, sino que ambas formas verbales señalan si el periodo de tiempo en que la acción se produce incluye o no el presente gramatical" (33). Sin embargo, existen diferencias dialectales entre España y América en la selección del PPS o del PPC.

Moreno de Alba (1978), por su parte, sostiene que "muchas de las expresiones que en el español peninsular se dan en antepresente [PPC], se manifiestan en el español mexicano en pretérito [PPS], limitando así el uso del antepresente [PPC]" (68). En el español de Castilla, "la característica principal del antepresente [PPC] es su valor temporal, su aproximación al presente gramatical (...) si la acción tuvo su perfección en el presente ampliado aparece el antepresente". Igualmente, el predominio del PPC sobre el PPS del español peninsular ha sido investigado tanto en ciertas zonas de levante (Alicante) (Schwenter 1994), como en el norte de España (Bilbao) (Burgo 2010), no solo en el contexto 'hodiernal', sino además en el 'prehodiernal'. Siguiendo la definición de Dahl (1985:125), el término 'prehodiernal' (en adelante PREH) se refiere a situaciones en las que las acciones expresadas por el verbo han ocurrido antes del día en que se produce la comunicación ( $<$ Lat. pre ('antes') + hodie ('hoy')), por ejemplo, 'Ayer / la semana pasada / el año pasado he ido de compras'. En estos casos, el complemento temporal PREH expresa una acción indicada por el predicado sin coincidir con el día de la comunicación, ya que resulta anterior al mismo. 
El evento 'hodiernal' (en adelante HOD), en cambio, hace referencia a la unidad de tiempo continua hasta el presente, esto es, el mismo día del acto de comunicación ( $<$ Lat. hodie ('hoy')), por ejemplo, 'Hoy / esta semana / este mes / este año he ido de compras'. Sin embargo, estudios similares llevados a cabo en las Islas Canarias (Herrera y Medina 1994; Serrano 1998; Piñero 2000) encuentran un aumento de preferencia por PPC, en vez del PPS. Por otra parte, Zamora Vicente (1967) deja constancia de que en Andalucía occidental (especialmente, Cádiz y Málaga) el PPC se apropia del papel del PPS. DeMello (1994), por su parte, identifica el habla de Sevilla como prototípica provincia en la que el empleo del PPC se hace para indicar una acción limitada al pasado, por ejemplo, "Ayer me he encontrado a uno en la calle" (612). Aunque DeMello observa que en un alto número de sus resultados el evento al que se refiere el hablante es reciente, por ejemplo, "Eso lo he visto hace poco", muchos de los casos en los que la relevancia del presente se relaciona con el pasado no se da por proximidad temporal, sino de forma más subjetiva: por razones de afectividad muchas veces difíciles de reconocer. Prueba de ello es el uso del PPC en un alto porcentaje con verbos en primera persona singular o plural.

En lo que respecta al español de México, Moreno de Alba (1978) y Lope Blanch (1972) coinciden en afirmar que la diferencia entre PPS y PPC se debe a matices aspectuales, esto es, siempre que un fenómeno se conciba como concluido, se usa el PPS, por cuanto este tiene un valor puntual, o semelfactivo, y perfectivo. El PPC, en cambio, implica una continuidad de la acción, con valor durativo o reiterativo.

Schwenter y Torres Cacoullos (2008) examinan exhaustivamente los contextos lingüísticos que favorecen el uso del PPC en España y en México, en cuanto a la referencia temporal (HOD, PREH, específica, indeterminada o irrelevante), el tipo de verbo (durativo o puntual), la presencia de un adverbio temporal, el número del sustantivo (plural o singular), la referencia temporal (HOD o PREH) y la presencia de la palabra ya. Este estudio no tiene en cuenta la persona gramatical, la polaridad ni el tipo de cláusula, por cuanto otros factores interfieren con ellos. Por ejemplo, la segunda persona aparecerá con más frecuencia en oraciones interrogativas necesariamente. Tanto en España como en México la referencia temporal irrelevante favorece el uso del PPC sobre el PPS, la referencia temporal tanto en México como en España es superior cuando no es relevante para la conexión con el presente está implícita. En ambos dialectos, el adverbio temporal y la palabra ya favorecen el PPC en cuanto su relación con el punto de referencia presente subyace al significado del PPC, incluso cuando en España tiene otros rasgos semánticos. En la variedad mexicana, por su parte, el PPC es favorecido por preguntas absolutas (también llamadas preguntas polares o preguntas de sí/ 
no) y cláusulas relativas. Asimismo, mientras los verbos durativos motivan el uso del PPC, en el dialecto español, en cambio, este factor no resultó significativo, lo cual es consecuente con la previa literatura anteriormente comentada. Para los autores, esto es prueba de que el PPC continúa en un proceso de gramaticalización ${ }^{1}$ en España, de modo que está perdiendo su sentido aspectual para convertirse en una marca general de pasado.

Puestas estas consideraciones en un contexto diacrónico, suscita la pregunta de cómo se relaciona el proceso evolutivo del PPC con la diferenciación dialectal arriba mencionada. Si nos remontamos al origen, hay que resaltar que el PPS y PPC tienen arranque en el latín clásico, en el que se distinguía una triple oposición: 'perfecto' (feci), 'futuro perfecto' (fecero) y 'pluscuamperfecto' (feceram). Por entonces, feci tenía dos valores: PPS y PPC. En el decurso del desarrollo al latín vulgar se introduce una nueva forma verbal: habeo factum (de donde proviene he hecho en español). Este nuevo paradigma verbal originó un proceso de gramaticalización, permitiéndose una expresión de un valor tanto aspectual como temporal (Harris 1982: 46-47). Tras el análisis de las lenguas romances contemporáneas, el autor distingue cuatro patrones sincrónicos para el uso de las dos categorías PPS y PPC:

El primer estadio es el punto de inicio común en el que el PPS (feci) se emplea primordialmente para marcar la relevancia del presente de un hecho que tuvo lugar, comenzó a tener lugar o no pudo tener lugar en el pasado, y el PPC (habeo factum) se restringe a presentar estados resultantes de acciones pasadas, pero no se usa para describir situaciones pasadas o situaciones pasadas recientes (Harris 1982: 49). Este último caso corresponde al perfecto de resultado, según Comrie (1976), que se refiere a una situación que es el resultado de una acción pasada (56). Este patrón puede encontrarse en dialectos sureños del italiano en Calabria y Sicilia; por ejemplo, en el calabrés, citando a Rohlfs (1966-1969), Harris (1982: 50) contrasta ' $m i$ scrissi' (él me ha escrito / me escribió) con 'non m'a scrivutu' (él no me ha escrito), en los cuales respectivamente se observa 'una ocasión sin especificar' (el hecho de escribir en algún momento) versus 'una acción sin experimentar' (no existen noticias de él).

Una de las definiciones de 'gramaticalización' ampliamente aceptada corresponde a la aportación de Traugott y König (1991), al concebirla como "the dynamic, unidirectional historical process whereby lexical items in the course of time acquire a new status as grammatical, morphosyntactic forms, and in the process come to code relations that either were not coded before or were coded differently" (p. 199). 
En la etapa II, el PPS ( $f e c i$ ) se refiere a acciones pasadas, incluyendo eventos pasados recientes y hechos durante un periodo de tiempo en progreso. Por otra parte, el PPC (habeo factum) comienza a tener relevancia del presente en contextos específicos, pero se restringe a un pasado reciente o persistente de aspecto durativo o iterativo (Harris 1982: 49). Conforme Comrie_(1976), éste corresponde al perfecto de situación persistente que describe "a situation that started in the past but continues (persists) into the present" (60). Ejemplos de este patrón pueden encontrarse en gallego, portugués y muchas variedades del español de Hispanoamérica, de modo que el español de México se encontraría en esta fase. Casos como 'no vino hoy' en vez de 'no ha venido hoy' o '¿qué pasó?' por ‘¿Qué ha pasado?' son ejemplos expuestos por Harris (1982: 53).

En el tercer estadio, el PPS (feci) se restringe a susodichas funciones, mientras que el PPC (habeo factum) asume el valor tradicional de PPC con relevancia en el presente. Según Harris (1982), a este tipo corresponden las variedades del español del norte de España (Aragón, Navarra). Igualmente, Escobar (1997: 860) apunta que en variedades andinas de Perú, que están en contacto con quechua, se hallan en esta etapa. Un ejemplo que presenta Alarcos (1947: 119), citado por Harris (1982: 55), se ilustra con ‘¿Cuándo ha llegao [sic] usted?' en que la acción, para el que pregunta, se ha producido necesariamente en un momento indeterminado del periodo comprendido entre un punto del pasado y el momento presente en que se habla.

Por último, el estadio IV experimenta la desaparición del contraste semántico entre el PPS (feci) y PPC (habeo factum). Mientras el PPS se restringe a un registro formal, el PPC (habeo factum) se emplea para funciones pretéritas y perfectivas (Harris 1982: 50). Ejemplos de lenguas en esta etapa corresponden al francés estándar, al italiano norteño y al rumano estándar.

Mientras que en algunas lenguas romances, como el francés y algunas variedades del italiano, se ha observado una evolución hasta el estadio IV, en el cual el PPC se hace idéntico al PPS y, generalmente, lo remplaza, el español estándar peninsular ha llegado solo al tercer estadio: el PPC no se apropia del papel del PPS, es decir, una acción pasada con relevancia del presente, pero sin señalar duración, repetición, etc. El proceso que sufre el verbo haber es el de gramaticalización. Comienza con un sentido resultativo, esto es, en el que haber designa un estado que es consecuencia de una acción, expresada ésta en un participio, de verbo reflexivo, concordante con un acusativo; en castellano, el verbo haber consumó su proceso de gramaticalización en el siglo XV, según se evidencia en el hecho de que para esta época deja de concordar el participio, el cual ya puede seleccionar verbos intransitivos sin restricciones de uso (Romani 2006). Schwenter y 
Torres Cacoullos (2008), por su parte, sugieren que en el español peninsular el proceso de gramaticalización continúa, pues el PPC pierde continuamente su sentido aspectual para convertirse de manera paulatina en una marca principalmente temporal.

Copple (2011) estudia los factores lingüísticos que influyen en la gramaticalización del PPC en España en tres períodos temporales (siglos XV, XVII y XIX). Los factores que tiene en cuenta son la referencia temporal (irrelevante, indeterminada, HOD o PREH), la expresión del sujeto, el tipo de verbo (atélico, realización y logro) y el tipo de cláusula (interrogativa o no interrogativa). La autora observa una tendencia al aumento de la referencia temporal irrelevante del siglo XV, con un peso de 0.54 , al XVII, con un peso de 0.83 , que se mantiene hasta el siglo XIX. El tipo de verbo tiene una distribución homogénea en todas las formas y es significativo solo en los siglos XV y XIX, dejando de serlo en el siglo XVII. Esta volubilidad del sistema verbal puede estar relacionado con el hecho de que el siglo XX, como señalan Schwenter y Torres Cacoullos (2008), no es significativo en España y en su historia tampoco se ha marcado aspectualmente el PPC. Del mismo modo existe una distribución homogénea significativa en oraciones interrogativas y no interrogativas para el siglo XV, pero esta significancia se pierde en el resto de los períodos.

Rodríguez Louro (2009) estudia un corpus del español argentino que divide en tres períodos (1810-1898; 1910-1970; 1982-2007) y del que tiene en cuenta los siguientes factores: adverbio temporal (ausencia, de proximidad $\mathrm{y}$ frecuencia u otro), persona gramatical (primera persona y un grupo de segunda y tercera personas), tipo de cláusula (principal y subordinada) y número del objeto (singular y plural). En el primer período (1810-1898) solamente el factor referido al adverbio temporal resulta significativo, con una preponderancia de 0.57 cuando éste se halla ausente. En el segundo período (1910-1970), el factor de mayor amplitud es el tipo de cláusula, con una mayor presencia de PPC en cláusulas subordinadas, lo que es consecuente con lo que Schwenter y Torres Cacoullos (2008) encontraran solo para el caso de México en oraciones de relativo. Objetos de número plural también tienen preponderancia, relacionado con la función continuativa del PPC, como también lo observan Schwenter y Torres Cacoullos. Los demás factores no muestran ser significativos en este período. En el último período (1982-2007), la presencia del adverbio temporal vuelve a resultar relevante a favor de los verbos de proximidad y de frecuencia. El número del objeto sigue siendo significativo en este período en los nombres plurales. La novedad es que los verbos atélicos ya favorecen el PPC, consecuente con el sentido durativo que Schwenter y Torres Cacoullos (2008) observan para el español de México. 
Estudios de tipo de diacrónico como los de Copple (2011) y Rodríguez Louro (2009) aportan un conocimiento muy valioso para la comprensión del proceso de cambio del PPC. Por un lado, ponen de relevancia que el PPC desempeña desde tiempos remotos la función de anclar un evento del pasado con la situación presente de quien habla, lo que se mantiene hasta el siglo XIX y XX tanto en América como en España. Sin embargo, en América adquiere un sentido aspectual durativo en el siglo XX, mientras que en España parece ganar terreno el sentido general de pasado, lo que implica un proceso de gramaticalización. El problema es que otros rasgos semánticos, como la persona gramatical, no han recibido suficiente atención y podrían revelar un proceso inverso: una resemantización del PPC a contextos de mayor subjetividad, como lo sugieren Company Company (2002: 63) y DeMello (1994) para el caso del español andaluz. Estudios de tipo histórico tienen la potencialidad de contribuir a una mejor comprensión de los fenómenos de variación y cambio, de modo que aquí nos hemos centrado en examinar un corte sincrónico del pasado para sopesar y comparar la antigüedad del fenómeno con una mirada dialectal contrastiva. A diferencia de las investigaciones de esta naturaleza ocupadas de áreas dialectales específicas, defendemos la precisión que podría ofrecer una mirada integradora del español peninsular y americano.

\section{METODOLOGÍA}

\subsection{CORPUS}

Nuestro corpus está compuesto por textos dramáticos y/o epistolares escritos a lo largo del siglo XIX. Por una parte, contamos con Alfredo (1835) que es un drama trágico en 5 actos y escrito por Joaquín-Francisco Pacheco, como texto representativo del corpus de España; por otra parte, una serie de textos (i.e., cartas privadas, testimonios en juicio, y memoriales y peticiones) recogidos en la compilación antológica Documentos Lingüísticos de la Nueva España (DLNE) (1994) por Concepción Company Company (1994) conforma el corpus del español de México.

A pesar de ser consciente de las diferencias existentes entre el lenguaje oral y escrito, la elección de estos textos como fuentes primarias e ilustrativas del lenguaje vernáculo del siglo XIX obedece, como principales razones, a que (i) el teatro se caracteriza por ser un escrito intencionado para su 
representación empleando una forma del lenguaje natural y, por consiguiente, más cercano al vernáculo (si, por ejemplo, se contrasta con otros géneros literarios como la poesía o la narrativa, donde el uso del lenguaje puede ser manipulado por el escritor por diferentes licencias literarias), y a que (ii) las cartas someramente informales destacan, lingüísticamente hablando, por su carácter coloquial, al igual que sobresalen, formalmente hablando, por una escrupulosa trascripción. En palabras de Company Company (1992), el volumen DLNE tiene como fin que "el lingüista interesado en historia de la lengua cuente con una herramienta útil para poder investigar y rastrear diacrónicamente distintos aspectos de la evolución del español de México" (802), lo cual responde a nuestro interés principal.

\subsection{DISEÑO Y CODIFICACIÓN DE LA MUESTRA}

Con objeto de que la estadística refleje el resultado de un corpus homogéneo, analizamos obras con un similar número de ocurrencias, es decir, 280 casos de España y 250 del texto de México. El total de muestras de aparición de las formas verbales analizadas es de 530, las cuales extraemos dentro del contexto oracional en el que aparecen. Procesamos todo el material a través del programa estadístico GoldVarb (Rand y Sankoff 1990), el cual, siendo un programa de análisis múltiple, está "deliberately designed to handle the kind of data obtained in studies of variation" (Bayley 2002: 124) y, tal y como muestran inéditas investigaciones, está comprobado que es "the most efficient and accurate tool in analyzing the multiple factors conditioning variant choice" (Aaron 2006: 54). Para ello, previamente se establece una codificación compatible con las reglas del programa. De este modo, la variable de aplicación es el PPC, la cual comparamos con el PPS.

Las variables lingüísticas independientes tenidas en cuenta a la hora de codificar los datos suman un total de seis grupos de factores:

(1) En primer lugar, consideramos si la persona gramatical del verbo $\left(1^{\mathrm{a}}\right.$, $2^{\mathrm{a}}$ y $3^{\mathrm{a}}$ persona singular/plural) depende o no de la elección temporal.

(2) La presencia de elementos temporales o no presencia (Ø: sin adverbio explícito), mediante un sintagma adverbial. Si se da la presencia de algún elemento temporal objetivo, se tiene en cuenta si éste es (a) hodiernal (dentro de las 24 horas del día), (b) hesternal (ayer), (c) anual (dentro del mismo año), (d) preanual (anterior al año pasado), o (e) inespecífico. Hay que apuntar que en un principio, los subfactores del sintagma adverbial eran (a) Ø (sin adverbio explícito), (b) hodiernal (dentro de las 24 horas del día), (c) hesternal (ayer), (d) anual (dentro 
del mismo año), (e) preanual (anterior al año pasado) y (f) inespecífico. Sin embargo, al observar que esta clasificación no favorecía los primeros resultados, una vez realizada la recodificación, optamos por reducirla a (a) Ø (sin adverbio explícito), (b) pre-anual (hace más de un año) y (c) anual (dentro del mismo año).

(3) Con respecto al tipo de enunciado (polaridad), distinguimos entre afirmativo y negativo.

(4) Según la actitud del hablante, clasificamos cada caso en frases declarativas, interrogativas o exclamativas.

(5) También tuvimos en cuenta el tipo de cláusula, es decir, si la forma verbal (PPS o PPC) aparecía en una oración principal o subordinada.

(6) En cuanto a la clase de verbo, hicimos la siguiente distinción semántica del verbo: estado, actividad, consecución y cumplimiento. La mayoría de estudios en esta área se ha basado en una división inicialmente propuesta por Vendler (1967). Éste es un sistema compuesto por cuatro tipos de clases aspectuales o predicados: de estado (haber, gustar, ser), de actividad (beber, escribir), de consecución (beber un café, escribir un poema) y de cumplimiento (abrir, romper, salir). Estas categorías se distinguen en base a tres rasgos semánticos: [puntual], [télico] y [dinámico]. De este modo, los predicados de cumplimiento como romper son [+puntual] porque no tienen duración. Además son [+télico] porque tienen un punto inicial y un punto final inherentes y son [+dinámico], ya que requieren energía física o mental. Los predicados de consecución como beber un café, por su parte, son [-puntual], puesto que tienen duración, son [+télico] porque tienen un punto inicial y un punto final inherentes y son [+dinámico], ya que también requieren energía física o mental. Los predicados de actividad, como beber en Alfredo bebe café, son [-puntual], pues tienen duración y [-télico] porque no tienen un punto inicial y un punto final inherentes. Asimismo, son [+dinámico], puesto que necesitan energía para que ocurran. En último lugar, los predicados de estado como ser tienen valores negativos para los tres rasgos mencionados [-puntual], [-télico] y [-dinámico]. Los predicados de estado expresan duración, no tienen un punto inicial y un punto final inherentes y existen sin necesidad de aplicar energía. La Tabla 1, adaptada de Andersen y Shirai (1994) resume los rasgos mencionados arriba. 
Tabla 1. Rasgos correspondientes a las distintas clases aspectuales

\begin{tabular}{|l|c|c|c|c|}
\hline & Estado & Actividad & Consecución & Cumplimiento \\
\hline Puntual & - & - & - & + \\
\hline Télico & - & - & + & + \\
\hline Dinámico & - & + & + & + \\
\hline
\end{tabular}

\subsection{Cuestiones DE INVESTIGACIÓN E HIPÓTESIS}

De lo que se ha escrito e investigado sobre el PPC aún queda por resolver la cuestión sobre la divergencia entre España y México:

Pregunta $\mathrm{n}^{\circ} 1$ : ¿Cómo se diferencian dialectalmente España de México según la frecuencia de uso del PPC y el PPS?

Hipótesis: Ya en el siglo XIX, se encuentra una mayor frecuencia de uso del PPC frente al PPS, mientras que en México el PPC tiene una frecuencia mucho más baja. Esto revela que en el siglo XIX ya se estaban dando tendencias generalizadoras para el PPC en España como investigaciones previas han notado para el siglo XX.

Pregunta $\mathrm{n}^{\circ} 2:$ ¿Cuáles son los factores que motivan el uso del PPC frente al PPS en estos dialectos?

Hipótesis: En España, se espera que el PPC se prefiera para la primera persona como reflejo de una mayor subjetividad por parte del hablante, factor que no resultará significativo en México. Igualmente, el factor de sintagma adverbial es un excelente candidato para un posible predominio del PPC, con una preponderancia del hodiernal para el PPC, pero con unos valores igualmente altos para los demás tiempos, dado un cambio en marcha que tiende a extender las posibilidades temporales del PPC. Tanto en México como en España se espera que la polaridad negativa, las cláusulas subordinadas y las oraciones interrogativas favorezcan el uso del PPC, por cuanto estos contextos suponen un anclaje con el tiempo presente que sobrevive en el PPC del español europeo y americano. También esperamos en España una significancia del tipo de verbo, dado que el PPC supone un matiz expresivo especial, mas no aproximamos una predicción específica al respecto. 


\section{RESULTADOS Y DISCUSIÓN}

La Tabla 2 refleja la frecuencia del PPC para el PPS en España y México. Existe una frecuencia más alta de PPC en España que en México, lo que es comparable con resultados similares para el siglo XX, por lo que la diferencia dialectal puede decirse que es un fenómeno ya apreciable en el siglo XIX.

Tabla 2. Muestras de PPS y PPC de España y México

\begin{tabular}{|l|c|c|c|}
\cline { 2 - 4 } \multicolumn{1}{c|}{} & España & México & Total \\
\hline PPS & 80 & 208 & 288 \\
\hline PPC & 200 & 42 & 242 \\
\hline Total & 280 & 250 & 530 \\
\hline
\end{tabular}

Por otra parte, la Tabla 3 presenta los resultados del análisis multivariado realizados mediante el uso del programa VARBRUL en su versión GoldVarb. Aquí mostramos los factores lingüísticos que afectan el uso de PPC en comparación con el PPS en España (E).

Tabla 3. Factores lingüísticos examinados con relevancia de uso del PPC

(S. XIX): España

\begin{tabular}{|c|c|c|c|c|}
\hline Factor & $\%$ PPC & Probabilidad & $N$ & $\%$ datos \\
\hline Persona & & & & \\
\hline $1^{\text {a }}$ y 2 ${ }^{\text {p personas plural }}$ & 91 & 0.79 & 22 & 8 \\
\hline $2^{\text {a }}$ persona singular & 88 & 0.70 & 16 & 6 \\
\hline $1^{\text {a }}$ persona singular & 75 & 0.54 & 76 & 27 \\
\hline Ellos/as & 65 & 0.41 & 166 & 59 \\
\hline Amplitud & 38 & & & \\
\hline Polaridad & & & & \\
\hline Negativa & 85 & 0.69 & 41 & 15 \\
\hline Afirmativa & 68 & 0.47 & 239 & 85 \\
\hline Amplitud & 22 & & & \\
\hline Sintagma Adverbial & & & & \\
\hline Annual & 86 & {$[0.79]$} & 7 & 3 \\
\hline Pre-Annual & 69 & {$[0.50]$} & 78 & 27 \\
\hline
\end{tabular}




\begin{tabular}{|l|c|c|c|c|}
\hline$\varnothing$ & 71 & {$[0.49]^{2}$} & 195 & 70 \\
\hline Tipo de Frase & & & & \\
\hline Exclamativa & 79 & {$[0.67]$} & 14 & 5 \\
\hline Interrogativa & 77 & {$[0.50]$} & 39 & 14 \\
\hline Declarativa & 69 & {$[0.49]$} & 227 & 81 \\
\hline Tipo de Cláusula & & & & \\
\hline Subordinada & 69 & {$[0.50]$} & 74 & 26 \\
\hline Principal & 71 & {$[0.49]$} & 206 & 74 \\
\hline Clase de Verbo & & & & \\
\hline Actividad & 90 & {$[0.76]$} & 29 & 10 \\
\hline Consecución & 78 & {$[0.59]$} & 27 & 9 \\
\hline Estado & 65 & {$[0.44]$} & 46 & 15 \\
\hline Cumplimiento & 59 & {$[0.40]$} & 178 & 66 \\
\hline
\end{tabular}

Log likelihood $=-161.264 ; \mathrm{p}=0.038 ;$ Chi-square $/$ cell $=1.1192$

Probabilidad de Input: 0.725 (70.7\%), N=200/280²

Contrario a nuestras expectativas, parece que ninguno de los factores sobresale significativamente; es decir, se observa que el sintagma adverbial, el tipo de frase, el tipo de cláusula y la clase de verbo no alcanzan un significado estadístico que favorezca la preferencia por el uso del PPC frente al PPS en España. Solamente la preferencia del PPC en cláusulas de polaridad negativa se aproxima a nuestras expectativas. Enunciados de polaridad afirmativa o negativa llegan a alcanzar una amplitud 22 y el que cuenta con mayor probabilidad es la negativa con 0.69. Esto manifiesta una posibilidad de que la acción negada, la cual se esperaba que hubiera acontecido en el pasado, tenga lugar en un futuro. Sirvan de ejemplos los siguientes casos extraídos de nuestro corpus:

(1) El cielo no ha permitido (PPC: enunciado negativo) mi muerte..., ¡ojalá me la hubiese enviado, antes de veros como os encuentro hoy! (E: 275)

(2) He conocido (PPC: enunciado afirmativo) en Alemania un trovador inglés que tornaba de la Palestina... De él aprendí este romance. (E: 48)

2 El corchete [ ] indica que este efecto no alcanza significancia estadística. 
En (1), el hablante expresa que el cielo "no ha permitido" su muerte y que deseaba haber muerto antes de observar el estado de ignonimia del interactor del diálogo. Así pues, el contexto negativo afirma un evento que el pasado no ha dado lugar y que en el presente tiene relevancia por cuanto se espera se presente en el futuro.

En cuanto al factor de persona (el factor de mayor magnitud de efecto con una amplitud: 38 ), la $2^{\mathrm{a}}$ (vosotros/as) y $3^{\mathrm{a}}$ (ustedes) personas del plural favorecieron el uso del PPC con una probabilidad de 0.79 de tal uso:

(3) Lo habéis decidido (PPC: $2^{\mathrm{a}}$ persona plural), por fin... estáis resuelto a emprender esa peregrinación... (E: 273)

(4) (...) nos persigue todo el día; que no ha dejado de volar en derredor de nosotros, y que en vano han querido (PPC: $3^{\mathrm{a}}$ persona del plural) ahuyentarlos ballesteros... (E: 259)

Los resultados referentes a persona muestran un matiz diferente a lo que hemos planteado en nuestras hipótesis. Los factores más altos con respecto a persona están dados por: $1^{\mathrm{a}}$ y $2^{\mathrm{a}}$ personas del plural, que se reagruparon en una sola categoría por tener valores muy similares; le sigue $2^{\mathrm{a}}$ persona de singular, y luego $1^{\mathrm{a}}$ persona de singular, siendo el puntaje más bajo para la $3^{\mathrm{a}}$ persona, en este caso de plural, pues no surgieron datos respecto al singular. Lo que es común a los datos de mayor frecuencia es que aparece en contextos donde alguno de los hablantes está involucrado. Esto evidencia que el PPC no implica necesariamente una subjetividad, sino un mayor anclaje al contexto comunicativo materializado en alguno de los participantes de la interacción.

La significancia estadística en los otros contextos puede interpretarse como parte de ese proceso de desgaste semántico del PPC en España que ya se evidencia en el siglo XIX. Es decir, la elección del PPC no está condicionada por esos contextos: no depende de una circunstancia temporal notablemente marcada, pues ya está proyectado libremente a referirse al pasado sin restricción, aunque favorecida por aquellos casos en los que algunos de los interlocutores está involucrado. Esto nos lleva a corroborar que el anclaje del pasado con el presente no depende de la instancia temporal como tal, sino de las circunstancias de enunciación en cuanto afecta al enunciador o enunciatario.

Este estado de hallazgos nos lleva a esperar para el caso de México (M) una mayor restricción contextual respecto al uso del PPC, como en efecto ocurre y se muestra en la Tabla 4. 
Tabla 4. Factores lingüísticos examinados con relevancia de uso del PPC

(S. XIX): México

\begin{tabular}{|c|c|c|c|c|}
\hline Factor & $\% P P C$ & Probabilidad & $N$ & $\%$ datos \\
\hline \multicolumn{5}{|l|}{ Polaridad } \\
\hline Negativa & 38 & 0.76 & 23 & 9 \\
\hline Afirmativa & 15 & 0.47 & 227 & 92 \\
\hline Amplitud & 29 & & & \\
\hline \multicolumn{5}{|l|}{ Tipo de Frase } \\
\hline Interrogativa & 86 & 0.95 & 8 & 3 \\
\hline Declarativa & 15 & 0.47 & 242 & 97 \\
\hline Amplitud & 48 & & & \\
\hline \multicolumn{5}{|l|}{ Tipo de Cláusula } \\
\hline Subordinada & 30 & 0.71 & 109 & 44 \\
\hline Principal & 6 & 0.33 & 141 & 57 \\
\hline Amplitud & 38 & & & \\
\hline \multicolumn{5}{|l|}{ Clase de Verbo } \\
\hline Actividad & 38 & 0.80 & 22 & 9 \\
\hline Consecución & 42 & 0.76 & 25 & 10 \\
\hline Estado & 21 & 0.67 & 28 & 11 \\
\hline Cumplimiento & 10 & 0.40 & 175 & 71 \\
\hline Amplitud & 40 & & & \\
\hline \multicolumn{5}{|l|}{ Sintagma Adverbial } \\
\hline$\varnothing$ & 14 & {$[0.45]$} & 175 & 71 \\
\hline Pre-Annual & 46 & {$[0.77]$} & 23 & 9 \\
\hline Annual & 14 & {$[0.51]$} & 52 & 21 \\
\hline \multicolumn{5}{|l|}{ Persona } \\
\hline$\neq 2^{a}$ persona singular & 16 & {$[0.49]$} & 245 & 94 \\
\hline $2^{\mathrm{a}}$ persona singular & 50 & {$[0.79]$} & 5 & 2 \\
\hline
\end{tabular}

Log likelihood $=-82.458 ; p=0.025 ;$ Chi-square $/$ cell $=0.8699$

Probabilidad de Input: 0.105 (16.8\%), N=42/250 
En cuanto al factor de mayor dimensión de efecto en el corpus de México, es el de polaridad (amplitud: 29). Dentro de esta variable lingüística, el que favorece al PPC es la negación con una probabilidad de 0.76. La afirmativa, por el contrario, aun siendo significativa, solo alcanza 0.47 de probabilidad. Observemos (5) y (6) como ejemplos del uso del PPC en enunciados negativos y afirmativos respectivamente:

(5) Y que en todo lo de /12 arriba estaba bien escrita su declaracion y $/ 13$ que no lo ha dicho por odio. Prometio el secre /14to y lo firmó de su nombre con dicho señor $/ 15$ comissario. (M: 128)

(6) Y procuró tranquilizarla, diciendo que no era bruto, $/ 20$ que lo más que ha hecho (afirmativa) con su hermana era tocarla los $/ 21$ pechos y partes ocultas. (M: 199).

Este resultado coincide con el de España y constituye presumiblemente el uso más conservador del PPC. Sin embargo, no encontramos en la literatura previa una inclusión de este factor en las tablas estadísticas, de modo que una tarea pendiente es observar el comportamiento de la polaridad en épocas anteriores y posteriores.

El segundo factor con alta dimensión y con predominio al PPC es el de tipo de frase (amplitud: 48). Dentro de este factor, el que favorece al PPC es la interrogativa con una probabilidad de 0.95 . La declarativa, en cambio, aun siendo significativa, sólo alcanza 0.47 de probabilidad. En los ejemplos (7) y (8) puede apreciarse el comportamiento del PPC según el tipo de frase:

(7) y $/ 22$ que le dixo: "sacamelo a mí y hasme como yo te $/ 23$ hize". Lo que efectuado, se derramó el padre en sus $/ 24$ manos y le dixo: "¿con que tú nunca te has redamado (PPC: interrogativa)? /25 ¡Mira, esto es redamarse!" (M: 243)

(8) El teniente letrado, encargado de la yntendencia de /2 Veracruz, me ha dirigido un expediente de quejas de $/ 3$ doña Maria Antonia Leal, vecina de Altotonga y viu /4da del teniente retirado, don Pablo Josef Bello, por tropelias /5 que ha cometido (PPC: declarativa) contra ella el encargado de justicia / 6 de dicho pueblo, don Gabriel Sanfuentes. (M: 2).

La presencia de PPC en oraciones interrogativas es consecuente con el sentido de evento posible en el pasado que anotábamos con respecto a la negación, y cuya posibilidad perdura en el momento de la enunciación, como en (7). 
El tercer factor que favorece el uso de PPC es el tipo de cláusula (amplitud: 38). Dentro de éste, el que prima con mayor probabilidad es la subordinada (0.77), frente a la principal (0.33). Considérense los siguientes ejemplos:

(9) Y con el mo /11 tibo de que as /12 mandado (PPC: subordinada) que $/ 13$ binieran tus $/ 14$ alguasiles a $/ 15$ cojer un ma $\{172 \mathrm{v}$, columna izquierda\} cho de Francisco / 2 Anttonio se ynfie /3re que es empeño $/ 4$ tullo el que $/ 5$ éstos caigan /6 en manos de $/ 7$ sus enemigos (M: 18)

(10) El teniente letrado, encargado de la yntendencia de /2 Veracruz, me ha dirigido (PPC: principal) un expediente de quejas de $/ 3$ doña Maria Antonia Leal, vecina de Altotonga y viu /4da del teniente retirado, don Pablo Josef Bello, por tropelias /5 que ha cometido contra ella (M: 2).

En (9), por ejemplo, el PPC está presente en una frase subordinada al sintagma nominal "el motivo". Esto es consecuente con un mayor condicionamiento sintáctico del PPC en cuanto a su relación con una instancia gramatical previamente enunciada. Si bien en (10) indicamos que el PPC puede aparecer en cláusulas principales, la preferencia por la subordinación es una señal de que el PPC en México se presenta en un contexto de relatividad sintáctica. Aunque Schwenter y Torres-Cacoullos (2008) reagrupan este factor de otra manera, aquí obtenemos similares resultados. Por consiguiente, esta diferencia sigue ocurriendo en el español actual y se remonta al siglo XIX, pero aún queda abierta la pregunta sobre la datación del fenómeno en un enfoque dialectal contrastivo de tipo diacrónico.

Por último, en cuanto al cuarto factor que desde un punto de vista semántico resulta ser ligeramente llamativo, la clase de verbo (amplitud: 40) parece favorecer el uso del PPC. Los verbos de actividad (0.80), consecución $(0.76)$ y estativos $(0.67)$ se encuentran con mayor probabilidad; los verbos de cumplimiento (0.40), por su parte, muestran menor uso. Véanse los siguientes ejemplos:

(11) Que como catholico christiano que es advi /19erte el que declara, que él ha cantado (PPC: actividad) tambien $/ 20$ dos o tres ocasiones dicho canto del arriero (M: 11)

(12) Que assi mismo concibe /8 y ha concebido que dicho Gutierres ha cantado dicho /9 son (PPC: consecución) por jocosidad (M: 12)

(13) Haciendole precen /23te de que mi conducta no ha cido (PPC: estado) santa, que /24 es a lo que todos devemos anelar (M: 40) 
(14) Que el referido havra co $/ 8$ mo doce años en diversas concurrencias y delante $/ 9$ de muchos lo ha cantado pero en distinto sentido /10 que el que se le ha preguntado, pues lo que le ha $/ 11$ percibido en su canto es que un arriero encuen /12tra a otro y éste pregunta si se le ha muerto /13 (PPC: cumplimiento) un burro y que aquél le responde que se le mu /14rio el borrico, y que éste cargado de /15 bulas se las havia comido llenandose de indili /16gencias (M: 185).

Interpretamos este fenómeno como una preferencia del PPC para enunciar eventos que otros tipos de situaciones. Dicho con otras palabras, la necesidad de marcar eventos posibles (como en la negación/interrogación) o relativos a otros eventos o elementos (como en cláusulas subordinadas) es más evidente cuando el verbo de por sí no es informativo al respecto. Los estados poseen en su contenido léxico semántico un rasgo aspectual durativo, y los verbos de cumplimiento, por su parte, perfectivo. Este resultado es, también, consecuente con lo que encuentran Schwenter y Torres Cacoullos (2008) en el español actual.

\section{CONCLUSIONES}

De todo lo expuesto hasta ahora se deduce que, a pesar de la inestabilidad de frecuencia del PPC en ambos países, ya en el siglo XIX percibimos cierta preferencia en el uso de ambas formas verbales de pasado. Retomando la clásica teoría de Moreno de Alba (1978), "muchas de las expresiones que en el español peninsular se dan en antepresente [PPC], se manifiestan en el español mexicano en pretérito [PPS], limitando así el uso del antepresente [PPC]" (68). Igualmente, con esto no queremos decir que el PPC esté en desuso en México, sino que su "su función denotativa es diferente y "su campo de acción" es más reducido" (Moreno de Alba 1978: 68). Entonces, esto responde a nuestra primera pregunta de investigación, pues la frecuencia de uso del PPS y PPC no es paralela en ambos países.

Una comparación de los factores lingüísticos revela que, mientras que en España los factores significativos son simplemente persona ( $1^{\mathrm{a}}$ persona singular, $2^{\mathrm{a}}$ y $3^{\mathrm{a}}$ personas plural, ellos/as, y $2^{\mathrm{a}}$ persona singular) y polaridad (afirmativa y negativa), en México, por el contrario, encontramos que (i) la persona resulta ser el factor menos significativo, (ii) comparte con el corpus de España el factor de polaridad (como significativo) y el de 
sintagma adverbial (como insignificante), (iii) cuenta con otros factores que ligeramente favorecen el uso del PPC: el tipo de frase (declarativa, interrogativa, exclamativa), el tipo de cláusula (principal y subordinada) y la clase de verbo (estado, actividad, consecución y cumplimiento). Estas diferencias confirman que en México la tendencia a un rasgo aspectual de tipo durativo es un fenómeno ya apreciable en el siglo XIX, puesto que se siente la necesidad de marcar eventos y no otros tipos de verbos que en su contenido léxico semántico ya expresan duratividad. Además, en México este sentido es relevante en cláusulas interrogativas y subordinadas debido a una mayor relatividad del evento a otro en el que se inscribe su posibilidad de continuidad, contrario a lo que ocurre en España.

Por otra parte, ni en México ni en España observamos una acción del sintagma adverbial, aunque anticipamos que una codificación que tenga en cuenta adverbios como ya y todavía podría revelar otro tipo de resultados. En España, si bien el desgaste semántico en factores que no han resultado aquí significativos podría interpretarse como signo de un proceso de gramaticalización, la preponderancia del PPC en situaciones donde interviene uno de los participantes de la conversación puede verse como una resemantización. Dicho con otras palabras, el PPC pierde algunos de sus rasgos gramaticales tradicionales y adquiere un rasgo deíctico. En nuestro corpus este tipo de factor no ha resultado significativo en México, aunque la naturaleza de las ocurrencias identificadas no permitió examinarlo en todo su rigor.

Llegando a estas conclusiones, como sugerencias para la investigación futura, podemos decir que aún queda por realizarse una datación de los factores lingüísticos que intervienen en la evolución del PPC desde el siglo XV hasta el presente, teniendo en cuenta que la persona gramatical es un rasgo que no debe dejarse a un lado, aun a expensas de los problemas estadísticos que pueda suponer. En general, todavía se necesita un trabajo de dialectología contrastiva que abarque un corpus diacrónico más amplio, preferiblemente desde el siglo XV hasta el presente, para caracterizar mejor si los hallazgos de este trabajo son de tipo conservador o innovador. 


\section{REFERENCIAS BIBLIOGRÁFICAS}

\section{CORPUS}

Alfredo $(1835)$ = Joaquín Francisco Pacheco. Biblioteca Virtual: Miguel de Cervantes. DLNE = Concepción Company Company: Documentos Lingüisticos de la Nueva España (1525-1818) (Altiplano Central) (México: UNAM, 1994), siglo XIX.

\section{REFERENCIAS CITADAS}

Aaron, Jessica E. 2006. Variation and change in Spanish future temporal expression. Tesis doctoral. University of New Mexico.

Alarcos Llorach, Emilio. 1978. Estudios de gramática funcional del español. Madrid: Editorial Gredos.

1947. Perfecto simple y compuesto en español. Revista de Filología española XXXI: 108-139.

Andersen, Roger W. y Yasuhiro Shirai. 1994. Discourse Motivations for Some Cognitive Acquisition Principales. Studies in Second Language Acquisition 16 (2): 133-156.

BAyley, RoBert. 2002. The quantitative paradigm. En J.K. Chambers, Peter Trudgill y Natalie Schilling-Estes (eds.). The Handbook of Language Variation and Change, pp. 117-41. Oxford: Blackwell.

Burgo, Clara. 2010. A case of grammaticalization in the use of the perfect for the preterite in Bilbao Spanish. Studies in Hispanic and Lusophone Linguistics 3 (2): 301-328.

Company Company, Concepción. 2002. Gramaticalización y dialectología comparada. Una isoglosa sintáctico-semántica del español. DICENDA. Cuadernos de Filología Hispánica 20: $39-71$.

1992. Los documentos lingüísticos de la Nueva España. Algunos rasgos del habla de un panadero mexicano del siglo XVII. En Manuel Ariza (coord.), pp. 333-341.

Comrie, Bernard. 1976. Aspect. Cambridge: Cambridge University Press.

Copple, MARY. 2011. Tracking the constraints on a grammaticalizing perfect(ive). Language Variation and Change 21: 163-191.

DAht, Östen. 1985. Tense and Aspect systems. Oxford - New York: Blackwell Publishers.

DeMello, G. 1994. Pretérito compuesto para indicar acción con límite en el pasado: 'Ayer he visto a Juan'. Boletín de la Real Academia Española 74: 611-633.

Escobar, AnNa M. 1997. Contrastive and innovative uses of the present perfect and the preterite in Spanish in contact with Quechua. Hispania 80: 859-870.

Harris, Martin. 1982. The 'past simple' and 'present perfect' in Romance. En Martin Harris, Martin y Nigel Vincent (eds.). Studies in the Romance Verb, pp. 42-70. London: Croom Helm.

Hernández, José Esteban. 2006. Present Perfect for Preterit in Salvadoran Narratives: The Perfective Expansion into Narrative Discourse. En Nuria Sagarra y Almeida Jacqueline Toribio (eds.). Selected Proceedings of the 9th Hispanic Linguistics Symposium, pp. 297307. Somerville, MA: Cascadilla Proceedings Project.

Herrera Santana, Juana y Javier Medina López. (1994). Sobre los usos de las formas de perfecto en el español atlántico. Anuario de Letras 32: 291-301

Lope Blanch, Juan Miguel. 1972. Sobre el uso del pretérito en el español hablado de México. En Juan Miguel Lope Blanch (ed.). Estudios sobre el español de México. México City: Universidad Nacional Autónoma de México. 
Moreno De Alba, José G. 1978. Valores de las formas verbales en el español de México. México: Universidad Nacional Autónoma de México.

Piñero Piñero, Gracia. 2000. Perfecto simple y perfecto compuesto en la norma culta de Las Palmas de Gran Canaria. Madrid: Vervuert-Iberoamericana.

Rand, DaVid y David Sankoff. 1990. GoldVarb: A variable rule application for Macintosh. Version 2. Montréal: Centre de recherches mathématiques, Université de Montréal.

Rodríguez Louro, Celeste. 2009. Perfect evolution and change: A sociolinguistic study of the Preterit and Present Perfect usage in contemporary and early Argentina. Tesis doctoral. University of Melbourne.

ROHLFS, GERHARD. 1966-1969. Grammatica storica della lingua italiana e dei suoi dialetti. Turin: Einandi.

Romani, Patrizia. 2006. Tiempos de formación romance I. Los tiempos compuestos. En Concepción Company Company (ed.), Sintaxis histórica de la lengua española, pp. 241346. México: Fondo de Cultura Económica.

Schwenter, ScotT A. 1994. The grammaticalization of an anterior in progress: Evidence from a peninsular Spanish dialect. Studies in Language 18 (1): 71-111.

Schwenter, Scott A y Rena Torres Cacoullos. 2008. Defaults and indeterminacy in temporal grammaticalization: the 'perfect' road to perfective. Language Variation and Change 20: 1-39.

SeCo, Rafael. 1985. Manual de gramática española. Madrid: Aguilar.

Serrano, María José. 1998. Grammaticalization and change in progress: Present Perfect in Spanish. En Luis Silva-Villar y Javier Gutiérrez-Rexach (eds.). Perspectives in Spanish Linguistics, pp. 117-136. Ohio: The Ohio State University.

Traugott, ElizABETH Y EKKEHARD KöNIG. 1991. Semantics-pragmatics of grammaticalization revisited. En Elizabeth Traugott y Bernd Heine (eds.). Approaches to Grammaticalization, vol. 1, pp. 189-218. Amsterdam: Benjamins.

Vendler, Zeno. 1967. Linguistics in Philosophy. Ithaca, NY: Cornell University Press.

Zamora Vicente, Alonso. 1967. Dialectología española. Madrid: Editorial Gredos. 